\title{
RATE DISTORTION OPTIMAL BIT ALLOCATION FOR STEREO IMAGE CODING
}

\author{
Walid Hachicha $^{1}$, Mounir Kaaniche ${ }^{1}$, Azeddine Beghdadi ${ }^{1}$, Faouzi Alaya Cheikh ${ }^{2}$ \\ ${ }^{1}$ L2TI, Institut Galilée, Université Paris 13, Sorbonne Paris Cité, France \\ ${ }^{2}$ The Norwegian Colour and Visual Computing Lab, Gjøvik University College, Norway \\ walid.hachicha@univ-paris13.fr, mounir.kaaniche@univ-paris13.fr, azeddine.beghdadi@univ-paris13.fr, faouzi.cheikh@hig.no
}

\begin{abstract}
Many research works have been developed for stereo image compression purpose where most of them aims at encoding a reference image, a residual one and a disparity map. While the disparity field is often losslessly encoded, we are mainly interested in this paper in the bit allocation problem between the reference and residual images. Generally, the bit allocation is expressed as an optimization problem which involves the computation of the operational rate-distortion ( $\mathrm{R}$ D) functions for all the wavelet subbands and for different quantization steps. However, this strategy is computationally intensive. To solve this problem, we consider the uniform scalar quantization of the wavelet subbands of both images modeled by a Generalized Gaussian distribution. Thanks to recent approximations of the entropy and distortion functions, we develop an optimal and fast bit allocation method. The obtained results confirm the efficiency of the proposed bit allocation method in the context of stereo image coding.
\end{abstract}

Index Terms - Stereo image, compression, bit allocation, wavelets, rate-distortion.

\section{INTRODUCTION}

The demand for 3D sensors and display technologies has been increasing in different emerging applications such as 3D TV, 3D Digital cinema, immersive games and video conferencing [1]. Indeed, a promising way of providing a realistic 3D imaging is to use the stereoscopic approaches. The basic idea behind a stereoscopic imaging system consists of generating two views, called left and right images, by recording two slightly different views angles of the same scene. Thus, the price of adding the third dimension to the viewer/player is the doubling of the image data size compared to the monoscopic case. Therefore, it becomes necessary to design efficient stereo compression techniques for storing and transmitting the involved huge amounts of data.

To this end, different approaches have been developed over the last decades since the pioneer work of Lukacs [2]. Intuitively, the simplest way for compressing stereo image is the independent coding scheme where the left and right images are encoded separately by using existing still image coders. However, since these images correspond to the same scene and so present similar visual contents, it has been shown that more efficient joint coding schemes can be developed by taking into account the inter-image redundancies. More precisely, the common idea behind most of the existing methods involves the following steps [2,3]. Firstly, one image (for example the left one) is selected as a reference image. After that, the disparity map, representing the displacement field between the pixels of the right and left images, is often estimated using a block-based approach. Then, the right view, referred to as target image, is pre- dicted from the reference one using the estimated disparity map, and the difference between the original target image and the predicted one, called residual image, is generated. Finally, the reference and residual images as well as the disparity map are encoded. While the disparity map is often losslessly encoded using a DPCM technique, the reference and residual images can be encoded in different transform domains [4-7].

While these steps have been commonly used in most of the developed works, the main differences between these works is related to the three following issues. Indeed, the first category of works aim at proposing a sophisticated Disparity Estimation/Compensation (DE/DC) methods $[8,9]$. The second one gives more attention to the choice of the transformations applied to the images. Among them, we mention the Discrete Cosine Transform $[4,5]$ and the Discrete Wavelet Transform [6,7]. Finally, the third category focuses on the entropy coding of the transformed coefficients [10]. However, the bit allocation issue for stereo image coding has rarely been addressed in the literature. Generally, the bit allocation process is based on Rate-Distortion (R-D) theory and aims at minimizing the distortion subject to a constraint on the available bitrate. We should note here that the problem of bit allocation has already been investigated in image and video compression standards [11]. Moreover, this problem has been studied in the context of Multi-view image coding in a depth-image-based-rendering framework $[12,13]$. In the context of stereo image coding, there is only few research works devoted to bit allocation between the two views. Among them, Woo and Ortega proposed in [14] a dependent bit allocation scheme between the left and residual images by using dynamic programming technique. However, in most of the developed stereo image coding schemes, the bit allocation problem is often solved by resorting to an empirical approach. In other words, for a given budget of bits, the reference and residual images are firstly encoded/decoded at different possible couple of bitrates. Then, the configuration yielding the minimum distortion value is retained as the optimal solution. However, such strategy of bit allocation is computationally intensive.

The objective of this paper is to design an efficient bit allocation technique for optimal stereo image coding. More specifically, thanks to recent approximation formulas of the entropy and distortion functions [15] and based on the Lagrangian optimization tool, explicit expression of the system of equations that satisfies the Lagrangian multiplier can be derived, which allows us to obtain a fast and accurate bit allocation strategy.

The remainder of this paper is organized as follows. In Section 2, we present the source and quantization models adopted in this work. Then, the proposed bit allocation method is described in Section 3. Finally, the efficiency of the proposed method in terms of ratedistortion criterion is shown in Section 4 and some conclusions are drawn in Section 5. 


\section{SOURCE AND QUANTIZATION MODELS}

Let $I^{(l)}$ and $I^{(e)}$ be the reference and residual images to be encoded. This is achieved by applying a wavelet transform which has been retained in the most recent compression techniques such as the JPEG2000 standard [16]. Thus, the sources to be quantized correspond to the $J$ subbands of the left and residual images denoted by $I_{j}^{(l)}$ and $I_{j}^{(e)}$ with $j \in\{1, \ldots, J\}$. Therefore, for a given subband $j$, the resulting wavelet coefficients can be well modeled by a Generalized Gaussian (GG) distribution whose probability density function is defined by:

$$
\forall \xi \in \mathbb{R}, \quad \tilde{f}_{j}^{(v)}(\xi)=\frac{\beta_{j}^{(v)}\left(\omega_{j}^{(v)}\right)^{1 / \beta_{j}^{(v)}}}{2 \Gamma\left(1 / \beta_{j}^{(v)}\right)} e^{-\omega_{j}^{(v)}|\xi|^{\beta_{j}^{(v)}}}
$$

where the superscript $v \in\{l, e\}$ is used to distinguish between the left view and the residual one, $\Gamma$ is the gamma function, $\omega_{j}^{(v)}$ and $\beta_{j}^{(v)}$ are respectively the scale and shape parameters which can be estimated using the method of moments or maximum likelihood techniques [17].

During the quantization procedure, a uniform scalar quantizer with a quantization step $q_{j}^{(v)}$ and a deadzone of size $\left(2 \tau_{j}^{(v)}-1\right) q_{j}^{(v)}$ where $\tau_{j}^{(v)}>\frac{1}{2}$, is employed to quantize all the wavelet subbands $I_{j}^{(l)}$ and $I_{j}^{(e)}$. Note that such a quantizer is often used in wavelet-based image compression schemes [16]. Thus, for each input coefficient, $I_{j, s}^{(v)}$ where $s$ denotes the spatial position, the output of the quantizer $\bar{I}_{j, s}^{(v)}$ is expressed as

$$
\bar{I}_{j, s}^{(v)}=0, \quad \text { if }\left|\bar{I}_{j, s}^{(v)}\right|<\left(\tau_{j}^{(v)}-\frac{1}{2}\right) q_{j}^{(v)}, \quad \text { where } \tau_{j}^{(v)}>1 / 2
$$

and, for all $i \in \mathbb{Z}, \bar{I}_{j, s}^{(v)}=r_{i, j}^{(v)}$,

$$
\text { (if }\left(\tau_{j}^{(v)}+i-\frac{3}{2}\right) q_{j}^{(v)} \leq \bar{I}_{j, s}^{(v)}<\left(\tau_{j}^{(v)}+i-\frac{1}{2}\right) q_{j}^{(v)} \text { and } i \geq 1 \text { ) or }
$$

(if $\left(-\tau_{j}^{(v)}+i+\frac{1}{2}\right) q_{j}^{(v)}<\bar{I}_{j, s}^{(v)} \leq\left(-\tau_{j}^{(v)}+i+\frac{3}{2}\right) q_{j}^{(v)}$ and $i \leq-1$ ), where the reconstruction levels are given by

$$
\forall i \geq 1, \quad r_{i, j}^{(v)}=-r_{-i, j}^{(v)}=\left(\tau_{j}^{(v)}+i-1\right) q_{j}^{(v)}
$$

Based on these source and quantization models, we address in what follows the bit allocation problem for encoding the quantized coefficients of the left and residual images.

\section{PROPOSED BIT ALLOCATION METHOD}

The proposed bit allocation method is based on the study of the rate and distortion functions. For this reason, we will firstly present accurate approximations of these functions for a quantized GG source.

\subsection{Rate and distortion approximation}

As generally considered in the design of R-D algorithms, we approximate the bitrate of the sources by a zero-order entropy of the quantized coefficients [18]. Thus, by assuming that the wavelet coefficients are modeled by a GG distribution, and based on recent approximation of the entropy of a quantized GG random variable [15], the entropy of the quantized subbands $\bar{I}_{j}^{(l)}$ and $\bar{I}_{j}^{(e)}$, denoted respectively by $H_{j}^{(l)}$ and $H_{j}^{(e)}$, can be approximated as follows: $\forall v \in\{l, e\}$,

$$
\begin{aligned}
& H_{j}^{(v)}\left(q_{j}^{(v)}\right)=-\mathrm{p}_{0, j}^{(v)} \ln \mathbf{p}_{0, j}^{(v)}-2 \mathbf{p}_{1, j}^{(v)} \ln \mathbf{p}_{1, j}^{(v)} \\
& +\left(h_{\beta_{j}^{(v)}}(1)-\ln \left(\left(\omega_{j}^{(v)}\right)^{1 / \beta_{j}^{(v)}} q_{j}^{(v)}\right)\right) \\
& \quad \times\left(1-Q_{1 / \beta_{j}^{(v)}}\left(\omega_{j}^{(v)}\left(\tau_{j}^{(v)}+\frac{1}{2}\right)^{\beta_{j}^{(v)}}\left(q_{j}^{(v)}\right)^{\beta_{j}^{(v)}}\right)\right) \\
& \quad+\frac{\omega_{j}^{(v)^{1 / \beta_{j}^{(v)}}}\left(\tau_{j}^{(v)}+\frac{1}{2}\right) q_{j}^{(v)}}{\Gamma\left(1 / \beta_{j}^{(v)}\right)} e^{-\omega_{j}^{(v)}\left(\tau_{j}^{(v)}+\frac{1}{2}\right)^{\beta_{j}^{(v)}}\left(q_{j}^{(v)}\right)^{\beta_{j}^{(v)}}} .
\end{aligned}
$$

where $\mathbf{p}_{0, j}^{(v)}, \mathbf{p}_{1, j}^{(v)}$ are the probabilities of the zero and the $r_{1, j}^{(v)}$ reconstruction levels [15] respectively, $h_{\beta_{j}^{(v)}}$ is the differential entropy of a GG distribution [19] and $Q_{a}$ with $a \in \mathbb{R}_{+}^{*}$ is the normalized incomplete Gamma function:

$$
\forall \xi \in \mathbb{R}, \quad Q_{a}(\xi)=\frac{1}{\Gamma(a)} \int_{0}^{\xi} \theta^{a-1} e^{-\theta} d \theta .
$$

Once the entropy is defined, the distortion function is evaluated through the $p_{j}$-th order moment of the quantization error. Here, we retain $p_{j}=2$ which represents the standard mean square error. While the distortion of a quantized GG random variable is given in [15] for a general order moment greater than or equal to 1 (i.e $p_{j} \geq 1$ ), the quadratic distortion (i.e $p_{j}=2$ ) of the quantized left and residual subbands, $D_{j}^{(l)}$ and $D_{j}^{(e)}$, becomes: $\forall v \in\{l, e\}$,

$D_{j}^{(v)}\left(q_{j}^{(v)}\right)=$

$$
\begin{aligned}
& \omega_{j}^{(v)-2 / \beta_{j}^{(v)}} \frac{\Gamma\left(3 / \beta_{j}^{(v)}\right)}{\Gamma\left(1 / \beta_{j}^{(v)}\right)} Q_{3 / \beta_{j}^{(v)}}\left(\omega_{j}^{(v)}\left(\left(\tau_{j}^{(v)}+\frac{1}{2}\right) q_{j}^{(v)}\right)^{\beta_{j}^{(v)}}\right) \\
& -2\left(\omega_{j}^{(v)}\right)^{-1 / \beta_{j}^{(v)}} \frac{\Gamma\left(2 / \beta_{j}^{(v)}\right)}{\Gamma\left(1 / \beta_{j}^{(v)}\right)} r_{1, j}^{(v)}\left(Q_{2 / \beta_{j}^{(v)}}\left(\omega_{j}^{(v)}\left(\left(\tau_{j}^{(v)}+\frac{1}{2}\right) q_{j}^{(v)}\right)^{\beta_{j}^{(v)}}\right)\right. \\
& \left.-Q_{2 / \beta_{j}^{(v)}}\left(\omega_{j}^{(v)}\left(\left(\tau_{j}^{(v)}-\frac{1}{2}\right) q_{j}^{(v)}\right)^{\beta_{j}^{(v)}}\right)\right) \\
& +\left(r_{1, j}^{(v)}\right)^{2}\left(Q_{1 / \beta_{j}^{(v)}}\left(\omega_{j}^{(v)}\left(\left(\tau_{j}^{(v)}+\frac{1}{2}\right) q_{j}^{(v)}\right)^{\beta_{j}^{(v)}}\right)\right. \\
& \left.-Q_{1 / \beta_{j}^{(v)}}\left(\omega_{j}^{(v)}\left(\left(\tau_{j}^{(v)}-\frac{1}{2}\right) q_{j}^{(v)}\right)^{\beta_{j}^{(v)}}\right)\right) \\
& +\frac{1}{12}\left(q_{j}^{(v)}\right)^{2}\left(1-Q_{1 / \beta_{j}^{(v)}}\left(\omega_{j}^{(v)}\left(\left(\tau_{j}^{(v)}+\frac{1}{2}\right) q_{j}^{(v)}\right)^{\beta_{j}^{(v)}}\right)\right)
\end{aligned}
$$

\subsection{Bit allocation approach}

In order to solve the inter-view bit allocation problem, our objective consists of finding the entropy values of the different subbands $\mathbf{H}$ $=\left(H_{1}^{(l)}, \ldots, H_{J}^{(l)}, H_{1}^{(e)}, \ldots, H_{J}^{(e)}\right) \in\left(\mathbb{R}^{+}\right)^{2 J}$ minimizing the average distortion subject to the constraint that the total bitrate is smaller than or equal to a given bitrate $R_{\max }$ :

$$
\begin{aligned}
\min \sum_{j=1}^{J} & \left(\rho_{j}^{(l)} D_{j}^{(l)}\left(H_{j}^{(l)}\right)+\rho_{j}^{(e)} D_{j}^{(e)}\left(H_{j}^{(e)}\right)\right) \\
& \text { subject to } \sum_{j=1}^{J} s_{j}\left(H_{j}^{(l)}+H_{j}^{(e)}\right)+R^{(d)} \leq R_{\max },
\end{aligned}
$$

where $R^{(d)}$ is the required bitrate for encoding losslessly the disparity map, $s_{j}$ corresponds to the fraction of total coefficients in the $j^{t h}$ subband, and $\left.\left(\rho_{j}^{(l)}, \rho_{j}^{(e)}\right) \in\right] 0,+\infty\left[^{2}\right.$ are two weights which 
account for the nonorthogonality of the wavelet transform. An appropriate choice of the weights $\left(\rho_{j}^{(l)}, \rho_{j}^{(e)}\right)_{1 \leq j \leq J}$ allows a good approximation of the distortion in the spatial domain.

The constrained minimization problem (7) is solved using the standard Lagrangian optimization technique. For any Lagrangian multiplier $\lambda<0$, the Lagrangian functional $\widetilde{\mathcal{J}}(\mathbf{H}, \lambda)$ is expressed as:

$$
\begin{aligned}
\widetilde{\mathcal{J}}(\mathbf{H}, \lambda)= & \sum_{j=1}^{J}\left(\rho_{j}^{(l)} D_{j}^{(l)}\left(H_{j}^{(l)}\right)+\rho_{j}^{(e)} D_{j}^{(e)}\left(H_{j}^{(e)}\right)\right) \\
& -\lambda\left(\sum_{j=1}^{J} s_{j}\left(H_{j}^{(l)}+H_{j}^{(e)}\right)+R^{(d)}-R_{\max }\right),
\end{aligned}
$$

Under the assumption of the differentiability, and after imposing the zero gradient condition, we find that the optimal entropy values are solutions of the following system:

$$
\forall j \in\{1, \ldots, J\}, \quad\left\{\begin{array}{l}
\frac{\rho_{j}^{(l)}}{s_{j}} \frac{\partial D_{j}^{(l)}}{\partial H_{j}^{(l)}}=\lambda \\
\frac{\rho_{j}^{(e)}}{s_{j}} \frac{\partial D_{j}^{(e)}}{\partial H_{j}^{(e)}}=\lambda
\end{array}\right.
$$

This equation shows that the optimal entropies correspond to the points having the same slope $\lambda$ on the R-D curves, $\left(H_{j}^{(l)}, \frac{\rho_{j}^{(l)}}{s_{j}} D_{j}^{(l)}\right)$ and $\left(H_{j}^{(e)}, \frac{\rho_{j}^{(e)}}{s_{j}} D_{j}^{(e)}\right)$, of both the left and residual images. Thus, it becomes necessary to compute the R-D curves of the different subbands. This step may be computationally heavy since a larger number of R-D operating points, that range from low to high bitrate, must be computed. However, it can lead to irregular functions which are neither convex nor differentiable. To deal with these problems, it has been proposed recently to perform the computation for a small number of R-D points belonging to the same convex hull, followed by a smooth spline interpolation [20].

Thanks to the developed R-D approximation, we propose in this paper to exploit the theoretical R-D approximations in order to avoid the interpolation and fitting procedures. Since the rate and distortion functions are expressed with respect to the quantization step $q_{j}^{(v)}$, Eq. (9) can be rewritten as:

$$
\forall j \in\{1, \ldots, J\}, \quad\left\{\begin{array}{l}
\frac{\rho_{j}^{(l)}}{s_{j}} \frac{\partial D_{j}^{(l)}}{\partial q_{j}^{(l)}}\left(\frac{\partial H_{j}^{(l)}}{\partial q_{j}^{(l)}}\right)^{-1}\left(q_{j}^{(l)}(\lambda)\right)=\lambda \\
\frac{\rho_{j}^{(e)}}{s_{j}} \frac{\partial D_{j}^{(e)}}{\partial q_{j}^{(e)}}\left(\frac{\partial H_{j}^{(e)}}{\partial q_{j}^{(e)}}\right)^{-1}\left(q_{j}^{(e)}(\lambda)\right)=\lambda
\end{array}\right.
$$

where, for each $v \in\{l, e\}$, the explicit expressions of the derivatives of $D_{j}^{(v)}$ and $H_{j}^{(v)}$ with respect to $q_{j}^{(v)}$ can be easily derived from (4) and (6). Thus, for a given value $\lambda$, the quantization steps $q_{j}^{(l)}(\lambda)$ and $q_{j}^{(e)}(\lambda)$ for the left and residual images can be obtained from (10). Finally, the rate allocation problem is solved by finding the optimal slope value $\lambda^{*}$ that satisfies:

$$
\sum_{j=1}^{J} s_{j}\left(H_{j}^{(l)}\left(q_{j}^{(l)}\left(\lambda^{*}\right)\right)+H_{j}^{(e)}\left(q_{j}^{(e)}\left(\lambda^{*}\right)\right)\right)=R_{\max }-R^{(d)}
$$

Simple algorithms such as Newton and bisection methods can be retained to find the optimal value $\lambda^{*}$. These algorithms converge in few iterations to the optimal solution if and only if the R-D curves are both convex and differentiable.

For this reason, it becomes necessary to check the convexity of the R-D curves resulting from our theoretical approximations of the rate and distortion functions given by (4) and (6). To this end, many experiments have been carried out on a large data set of stereo images. It is worth pointing out that the convexity property has been often satisfied for the most wavelet subbands, as it can be seen in the left side of Fig. 1. However, we have observed that it is not the case for some subbands. For example, the right side of Fig. 1 shows a particular case where $\frac{\partial D_{j}}{\partial H_{j}}$ is a non monotonic function. To deal with this

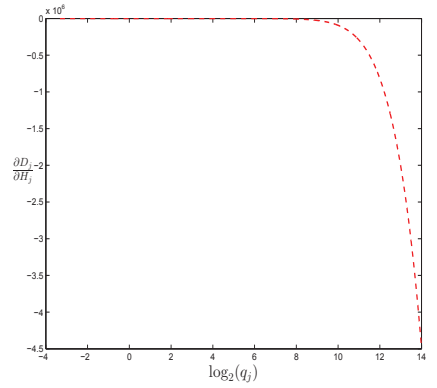

(a)

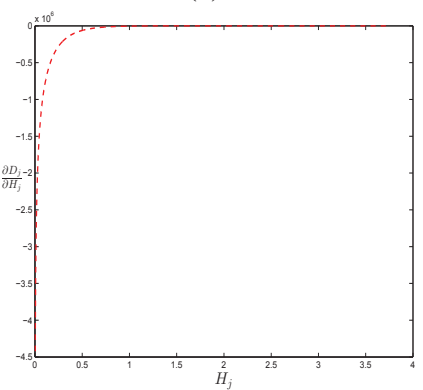

(c)

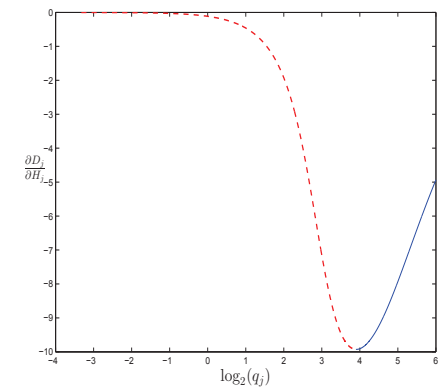

(b)

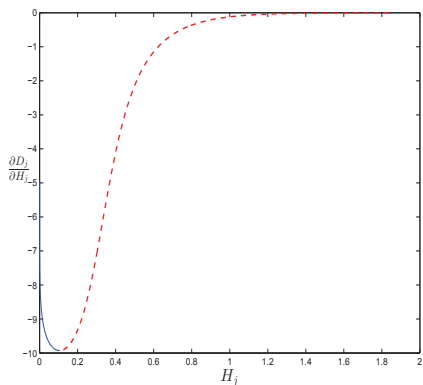

(d)
Fig. 1: In the top side (resp. bottom side), the first derivative function $\frac{\partial D_{j}}{\partial H_{j}}$ versus $\log _{2}\left(q_{j}\right)$ (resp. $H_{j}$ ) obtained with (left side): $\beta_{j}=0.5$ and $\omega_{j}=0.1$, (right side): $\beta_{j}=1.5$ and $\omega_{j}=0.1$.

problem, we have determined the inflexion point $\bar{q}_{j}^{(v)}$ of the curve by solving this equation:

$$
\frac{\partial^{2} D_{j}^{(v)}}{\partial^{2} H_{j}^{(v)}}=0 \Leftrightarrow \frac{\partial^{2} D_{j}^{(v)}}{\partial^{2} q_{j}^{(v)}} \frac{\partial H_{j}^{(v)}}{\partial q_{j}^{(v)}}-\frac{\partial^{2} H_{j}^{(v)}}{\partial^{2} q_{j}^{(v)}} \frac{\partial D_{j}^{(v)}}{\partial q_{j}^{(v)}}=0
$$

In this case, during the determination of $q_{j}^{(l)}(\lambda)$ and $q_{j}^{(e)}(\lambda)$ that satisfy Eq. (10), we are restricted to the search interval $\left.] 0, \bar{q}_{j}^{(v)}\right]$ with $v \in\{l, e\}$. It is important to note that, in this interval, we have observed that the possible entropy values range from low to high bitrate as shown in Fig. 1(d), which could not affect the optimality of the bit allocation method.

\section{EXPERIMENTAL RESULTS}

In order to evaluate the proposed bit allocation algorithm, we used different standard stereo images downloaded from some public stereovision datasets ${ }^{1}$ and ${ }^{2},{ }^{3}$. The disparity map is estimated based on the block-matching technique with a $8 \times 8$ block size, and then losslessly encoded by using an arithmetic coder. The 9/7 wavelet transform, selected for the lossy compression mode of JPEG2000 [16],

\footnotetext{
${ }^{1} \mathrm{http}: / /$ vision.middlebury.edu/stereo/

${ }^{2} \mathrm{http}: / / \mathrm{vasc} . \mathrm{ri} . \mathrm{cmu} . \mathrm{edu} / \mathrm{idb} / \mathrm{html} / \mathrm{stereo} / \mathrm{index} \cdot \mathrm{html}$

${ }^{3} \mathrm{http}: / / \mathrm{vasc} . \mathrm{ri} . \mathrm{cmu} . \mathrm{edu} / \mathrm{idb} / \mathrm{html} / \mathrm{jisct} /$
} 
has been retained to encode the reference and residual images. This decomposition is carried out over three resolution levels and the resulting wavelet coefficients have been encoded by using the entropy coder EBCOT. Note that the weights $\left(\rho_{j}^{(l)}, \rho_{j}^{(e)}\right)_{1 \leq j \leq J}$ for the wavelet subbands of the reference and residual images are computed by using the procedure described in [21].

The accuracy and the efficiency of the proposed bit allocation scheme is evaluated and compared with the optimal allocation strategy which is often used in the reported stereo image compression works. This strategy, denoted in the following by "Exhaustive search", considers firstly several configurations of rate allocation $\left(R^{(l)}, R^{(e)}\right)$, where $R^{(l)}+R^{(e)}=R_{\max }-R^{(d)}$, and $R^{(l)}, R^{(e)}$ and $R^{(d)}$ denote the bitrate of the left image, residual one and the disparity map, respectively. Then, the best solution corresponds to the couple $\left(R^{(l)}, R^{(e)}\right)$ leading to the minimum distortion evaluated through the Mean Square Error of the left image $\mathrm{MSE}^{(l)}$ and the right one $\mathrm{MSE}^{(r)}$ obtained after the decoding process. We have also tested another approach based on a prior given rate allocation. More precisely, $50 \%$ of the available bitrate is assigned to each view. In the following, this strategy will be designated by "Fixed". The reported results are given in terms of the average bitrate $R_{\mathrm{av}}$ and its corresponding PSNR measure:

$$
\begin{aligned}
& R_{\mathrm{av}}=\frac{R^{(l)}+R^{(e)}+R^{(d)}}{2}, \\
& \operatorname{PSNR}=10 \log _{10} \frac{255^{2}}{\left(\mathrm{MSE}^{(l)}+\mathrm{MSE}^{(r)}\right) / 2},
\end{aligned}
$$

Figure 2 illustrates the performance of the different considered bit allocation methods. It is clear that the R-D results of the fixed rate allocation strategy depends on the stereo data and leads to worse coding performance. Moreover, it can be noticed that the R-D curves obtained by the proposed method are very close to those resulting from the optimal allocation method based on the exhaustive search procedure.

For more convenience, we have also compared our proposed method to the exhaustive search one in terms of Bjontegaard metric, often used to measure the distance between two R-D curves [22]. The PSNR differences and the bitrate saving between these two approaches are provided in Table 1 for low, middle and high bitrates corresponding to the four target bitrate points $\{0.15,0.2,0.3,0.4\}$, $\{0.5,0.6,0.7,0.8\}$ and $\{0.9,1,1.1,1.2\}$ bpp, respectively. This table shows that the average of the PSNR differences between the R-D results are about $0.1-0.14 \mathrm{~dB}$.

While achieving R-D performance similar to that obtained with the exhaustive search allocation strategy, our proposed method reduces significantly the required execution time, as shown in Table 2. The simulations are carried out by using an Intel Core $2(3.2 \mathrm{GHz})$ computer with a Matlab implementation. It can be observed that the exhaustive search method is too computationally expensive whereas our proposed bit allocation method takes only few seconds. According to Table 2, the proposed method is about twenty times faster than the exhaustive search one.

\section{CONCLUSION}

In this work, we have addressed the bit allocation problem for stereo image compression. To this end, we proposed a fast and accurate bit allocation algorithm based on recent approximation of R-D functions. Experimental results show the effectiveness of the proposed bit allocation algorithm compared to the exhaustive search allocation procedure. In future work, the proposed bit allocation method could be extended to a multiview/video coding framework.
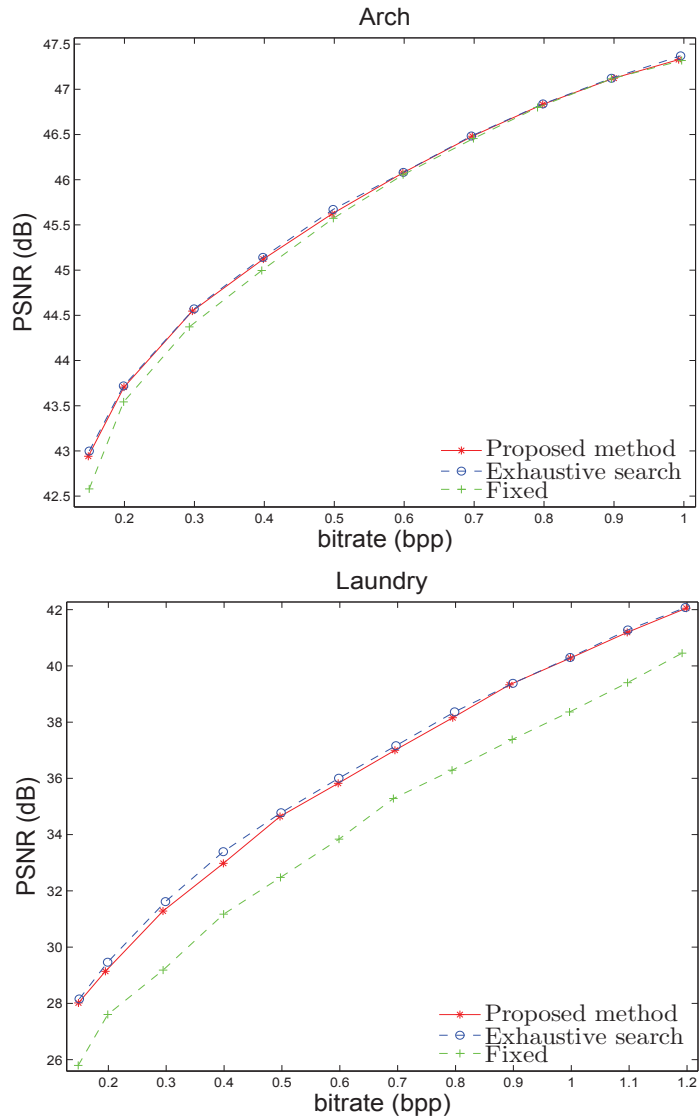

Fig. 2: Performance of the proposed bit allocation method w.r.t to the considered strategies for the images "Arch" and "Laundry".

\begin{tabular}{|c|c|c|c|c|c|c|}
\hline \multirow{2}{*}{ Image } & \multicolumn{3}{|c|}{ PSNR gain $(\mathrm{dB})$} & \multicolumn{3}{c|}{ Bitrate saving (\%) } \\
\cline { 2 - 7 } & low & middle & high & low & middle & high \\
\hline Houseof & -0.14 & -0.13 & -0.19 & 4.80 & 2.93 & 3.23 \\
\hline Pentagon & -0.11 & -0.08 & -0.09 & 3.71 & 1.73 & 1.62 \\
\hline Arch & -0.02 & -0.01 & -0.06 & 0.77 & 0.39 & 1.91 \\
\hline Ball & -0.03 & -0.01 & -0.18 & 1.64 & 0.19 & 6.24 \\
\hline Laundry & -0.25 & -0.16 & -0.04 & 4.69 & 2.03 & 0.42 \\
\hline Reindeer & -0.25 & -0.21 & -0.25 & 4.63 & 3.36 & 3.57 \\
\hline Average & -0.13 & -0.10 & -0.14 & 3.37 & 1.77 & 2.83 \\
\hline
\end{tabular}

Table 1: The average PSNR differences and the bitrate saving between the proposed and exhaustive search methods at low, middle and high bitrates.

\begin{tabular}{|c|c|c|c|}
\hline Image & Method & Encoding Time (s) & Ratio \\
\hline \multirow{2}{*}{ Pentagon } & Proposed & 3.02 & \multirow{2}{*}{24.90} \\
\cline { 2 - 3 } & Exhaustive search & 75.18 & \multirow{2}{*}{18.30} \\
\hline \multirow{2}{*}{ Laundry } & Proposed & 3.14 & \\
\cline { 2 - 4 } & Exhaustive search & 57.45 & \\
\hline
\end{tabular}

Table 2: Encoding time of the different approaches. 


\section{REFERENCES}

[1] I. Feldmann, W. Waizenegger, N. Atzpadin, and O. Schreer, "Real-time depth estimation for immersive 3D videoconferencing," in 3DTV-Conference: The True Vision-Capture, Transmission and Display of $3 D$ Video, Tampere, Finland, 2010, pp. 1-4.

[2] M. E. Lukacs, "Predictive coding of multi-viewpoint image sets," in IEEE International Conference on Acoustics Speech and Signal Processing, Tokyo, Japan, 1986, pp. 521-524.

[3] M. G. Perkins, "Data compression of stereopairs," IEEE Transactions on Communications, vol. 40, no. 4, pp. 684-696, 1992.

[4] W. Hachicha, A. Beghdadi, and F. A Cheikh, "1D directional DCT-based stereo residual compression," in European signal Processing Conference, Marrakech, Morocco, 2013.

[5] M. S. Moellenhoff and M. W. Maier, "Transform coding of stereo image residuals," IEEE Transactions on Image Processing, vol. 7, no. 6, pp. 804-812, June 1998.

[6] N. V. Boulgouris and M. G. Strintzis, "A family of waveletbased stereo image coders," IEEE Transactions on Circuits and Systems for Video Technology, vol. 12, no. 10, pp. 898903, 2002.

[7] M. Kaaniche, A. Benazza-Benyahia, B. Pesquet-Popescu, and J-C. Pesquet, "Vector lifting schemes for stereo image coding," IEEE Transactions on Image Processing, vol. 18, no. 11, pp. 2463-2475, 2009.

[8] W. Woo and A. Ortega, "Overlapped block disparity compensation with adaptive windows for stereo image coding," IEEE Transactions on Circuits and Systems for Video Technology, vol. 10, no. 2, pp. 194-200, 2000.

[9] L. F. R. Lucas, N. M. M. Rodrigues, E. A. B. da Silva, and S. M. M. de Faria, "Stereo image coding using dynamic template-matching prediction," in IEEE International Conference on Computer as a Tool, Lisbon, Portugal, 2011, pp. 1-4.

[10] J. N. Ellinas and M. S . Sangriotis, "Stereo image compression using wavelet coefficients morphology," Image and Vision Computing, vol. 22, no. 4, pp. 281-290, 2004.

[11] T. Wiegand, G. J. Sullivan, G. Bjontegaard, and A. Luthra, "Overview of the H. 264/AVC video coding standard," IEEE Transactions on Circuits and Systems for Video Technology, vol. 13, no. 7, pp. 560-576, 2003.

[12] G. Cheung, V. Velisavljevic, and A. Ortega, "On dependent bit allocation for multiview image coding with depth-image-based rendering," IEEE Transactions on Image Processing, vol. 20, no. 11, pp. 3179-3194, 2011.

[13] B. Rajaei, T. Maugey, H.-R. Pourreza, and P. Frossard, "Ratedistortion analysis of multiview coding in a DIBR framework," Annals of telecommunications, pp. 1-14, 2012.

[14] W. Woo and A. Ortega, "Optimal blockwise dependent quantization for stereo image coding," IEEE Transactions on Circuits and Systems for Video Technology, vol. 9, no. 6, pp. 861-867, 1999.

[15] M. Kaaniche, A. Fraysse, B. Pesquet-Popescu, and J.-C. Pesquet, "A bit allocation method for sparse source coding," IEEE Transactions on Image processing, vol. 23, no. 1, pp. 137-152, January 2014.
[16] D. Taubman and M. Marcellin, JPEG2000: Image Compression Fundamentals, Standards and Practice, Kluwer Academic Publishers, Norwell, MA, USA, 2001.

[17] M. N. Do and M. Vetterli, "Wavelet-based texture retrieval using generalized Gaussian density and Kullback-Leibler distance," IEEE Transactions on Image Processing, vol. 11, no. 2, pp. 146-158, February 2002.

[18] H. Gish and J. Pierce, "Asymptotically efficient quantizing," IEEE Transactions on Information Theory, vol. 14, no. 5, pp. 676-683, 1968.

[19] W. Szepanski, “ $\Delta$-entropy and rate distortion bounds for generalized Gaussian information sources and their application to image signals," Electronics Letters, vol. 16, no. 3, pp. 109-111, 1980.

[20] T. André, M. Cagnazzo, M. Antonini, and M. Barlaud, "JPEG2000-compatible scalable scheme for wavelet-based video coding," EURASIP Journal on Image and Video Processing, vol. 2007, 2007.

[21] S. Parrilli, M. Cagnazzo, and B. Pesquet-Popescu, "Distortion evaluation in transform domain for adaptive lifting schemes," in International Workshop on Multimedia Signal Processing, Cairns, Queensland, Australia, October 2008.

[22] G. Bjontegaard, "Calculation of average PSNR differences between RD curves," Tech. Rep., ITU SG16 VCEG-M33, Austin, TX, USA, April 2001. 